\title{
Application of Laser-Enhanced Ionization: Atomization Efficiency Determination
}

\author{
KING-DOW SU, KING-CHUEN LIN, * and WEI-TZOU LUH \\ Department of Chemistry, National Taiwan University, and Institute of Atomic and Molecular Sciences, Academia Sinica, \\ P.O. Box 23-166, Taipei, Taiwan 10764, Republic of China (K.-D.S., K.-C.L.); and Institute of Atomic and Molecular Sciences, \\ Academia Sinica, P.O. Box 23-166, Taipei, Taiwan 10764, Republic of China (W.-T.L.)
}

\begin{abstract}
We have demonstrated that the laser-enhanced ionization (LEI) technique can be used to determine the efficiency of atomization of metal elements in an atmospheric acetylene/air flame. We have derived a useful relation between the time-integrated LEI signal and the total free atom number density in a flame. We determine the efficiency of atomization of $\sim 0.13-0.37$ for the lithium element and of $\sim 1.0$ for the sodium element. Our results agree well with AA measurements reported previously. Index Headings: Atomic absorption spectroscopy; Flame spectroscopy.
\end{abstract}

\section{INTRODUCTION}

For analytical flame atomic spectrometry, various measurements of physical quantities are based on the free atom number density of an element in a flame. Thus the atomization efficiency ${ }^{1-8}$ (or the degree of atomization) of elements being analyzed is one of the crucial factors which govern the limit of detection. Previous measurements on the atomization efficiency of elements in flames have been performed only by using the atomic absorption method $^{2-7}$ or by using the atomic emission method. 8

However, during the last decade the laser-enhanced ionization (LEI) technique has been developed as a powerful tool for detecting trace metals on a sub-pg/mL scale with extremely high sensitivity and selectivity. ${ }^{9-15}$ The LEI technique has also been successfully used as an alternative detector for liquid chromatography ${ }^{16,17}$ and gas chromatography. ${ }^{18}$ On the basis of the Einstein relation, Lin et al. ${ }^{19}$ have applied the LEI technique to estimate the flame temperature after a quite involved procedure. Very recently, Su et al. ${ }^{20}$ have derived, alternatively, a more elaborate model for the LEI process which has been shown to be a more handy and precise method for determining the temperature of an atmospheric acetylene/ air flame.

In this paper, we have derived another useful scheme to apply the LEI technique for determining the efficiency of atomization of metal elements in a flame. In this scheme, rate equations for population densities at relevant atomic energy levels and the relevant ionic state of an atom have been solved. This approach results in a useful relation between the time-integrated LEI signal and the total number density of free atoms in a flame. We have applied this relation for determining the atomization efficiency of lithium and sodium in an acetylene/air flame. In the following, the theoretical treatment is first described, followed by a brief description of the

Received 3 March 1992; revision received 2 May 1992.

* Author to whom correspondence should be sent. experiments performed. Finally, the results obtained are presented and discussed.

\section{THEORY}

The atomization efficiency $\epsilon_{a}$ is defined as ${ }^{1}$

$$
\epsilon_{a}=n_{a} / n_{t}
$$

where $n_{a}$ is the number density of free atoms of an element present in the flame, and $n_{t}$ is the total number density of the same element actually nebulized. In the following discussion, we show that the number density $n_{a}$ can be determined via the LEI measurement.

The Free Atom Number Density $\boldsymbol{n}_{a}$ from the LEI Measurement. To deal with laser-enhanced ionization of atoms in a flame, one should consider both atomic $(1 \rightarrow 2)$ transitions and subsequent $(2 \rightarrow i)$ ionization processes. ${ }^{1,13,20-23}$ The schematic diagram for the atomic levels and ionic state needed in this work is shown in Fig. 1. This system may be described in terms of rate equations ${ }^{23}$

$$
\begin{aligned}
\frac{\mathrm{d} n_{1}}{\mathrm{~d} t}= & n_{2}\left(B_{21} \rho_{v}+A_{21}+K_{21}\right) \\
& -n_{1}\left(B_{12} \rho_{\nu}+K_{12}+K_{1 i}\right)+n_{i} K_{i 1} \\
\frac{\mathrm{d} n_{2}}{\mathrm{~d} t}= & n_{1}\left(B_{12} \rho_{v}+K_{12}\right) \\
& -n_{2}\left(B_{21} \rho_{v}+A_{21}+K_{21}+K_{2 i}\right)+n_{i} K_{i 2} \\
\frac{\mathrm{d} n_{i}}{\mathrm{~d} t}= & n_{1} K_{1 i}+n_{2} K_{2 i}-n_{i}\left(K_{i 1}+K_{i 2}\right)
\end{aligned}
$$

where $n_{1}, n_{2}$, and $n_{i}$ represent the number density at level 1 , level 2, and the ionic state, respectively; $A_{l m}$ and $B_{l m}$ the Einstein $A$ and $B$ coefficients; $\rho_{\nu}$ the laser spectral energy density; and $K_{r s}$ the collision rate coefficients. These rate equations can be approximated as ${ }^{20-23}$

$$
\begin{aligned}
\frac{\mathrm{d} n_{1}}{\mathrm{~d} t} & =n_{2}\left(B_{21} \rho_{v}+A_{21}+K_{21}\right)-n_{1} B_{12} \rho_{v} \\
\frac{\mathrm{d} n_{2}}{\mathrm{~d} t} & =n_{1} B_{12} \rho_{v}-n_{2}\left(B_{21} \rho_{v}+A_{21}+K_{21}+K_{2 i}\right) \\
\frac{\mathrm{d} n_{i}}{\mathrm{~d} t} & =n_{2} K_{2 i}
\end{aligned}
$$

where the collisional ionization $\left(n_{1} K_{1 i}\right)$ and collisional excitation $\left(n_{1} K_{12}\right)$ terms, having a smaller magnitude, and the recombination $\left(n_{i} K_{i 1}\right.$ and $\left.n_{i} K_{i 2}\right)$ term, occurring 
on a longer time scale, ${ }^{11}$ are individually neglected. These three coupled differential equations $(5,6$, and 7$)$ can be solved via the Laplace transform method. The results are

$$
\begin{aligned}
n_{1}(t)= & \frac{1}{x-y} n_{1}^{t h}\left[(x-a-c) e^{-x t}\right. \\
& \left.+(a+c-y) e^{-y t}\right] \\
n_{2}(t)= & \frac{b}{x-y} n_{1}^{t h}\left[-e^{-x t}+e^{-y t}\right] \\
n_{i}(t)= & \frac{1}{x-y} n_{1}^{t h}\left[x\left(1-e^{-y t}\right)\right. \\
& \left.-y\left(1-e^{-x t}\right)\right]
\end{aligned}
$$

where $a=B_{21} \rho_{\nu}+A_{21}+K_{21}, b=B_{12} \rho_{p}, c=K_{2 i}, x=\{a$ $\left.+b+c+\left[(a+b+c)^{2}-4 b c\right]^{1 / 2}\right\} / 2, y=\{a+b+c$ $\left.-\left[(a+b+c)^{2}-4 b c\right]^{1 / 2}\right\} / 2$; and $n_{1}^{\text {th }}$ is the thermal population density of the level 1 . Note that Eqs. 9 and 10 are respectively consistent with equations 9 and 8 of Omenetto et al. ${ }^{22}$ The experimental LEI current signal is related to the time derivative of the ion number density $n_{i}(t)$ by the relation ${ }^{22}$

$$
\begin{aligned}
& \frac{1}{G e \sigma \ell \gamma} \int_{0}^{\tau} V(t) \mathrm{d} t=\int_{0}^{t}\left(\frac{\mathrm{d} n_{i}(t)}{\mathrm{d} t}\right) \mathrm{d} t \\
& =\frac{1}{x-y} n_{1}^{\text {th }}\left\{x\left[1-\exp \left(-y \tau_{l}\right)\right]\right. \\
& \left.-y\left[1-\exp \left(-x \tau_{l}\right)\right]\right\}
\end{aligned}
$$

where $G$ is the gain of the current amplifier; $e$ the electron charge; $\sigma$ the cross section between the laser beam and the flame; $\ell$ the probing length, $\gamma$ the collection efficiency; $\tau$ the integration time interval; $V(t)$ the amplified voltage pulse of the time-resolved LEI signal; and $\tau_{l}$ the laser pulse duration. Note that this equation is the same as equation 11 of Omenetto et al. ${ }^{22}$ However, we will use the entire expression in this work without the approximation used previously. If the Boltzmann distribution law is locally obeyed in the flame, then the number density $n_{a}$ of free atoms and the number density $n_{1}^{\text {th }}$ would follow the relation

$$
n_{1}^{t h}=n_{a} \frac{g_{1}}{Q_{e}} e^{-\left(E_{1} / k T\right)}
$$

where $g_{1}$ is the degeneracy of the atomic level $1 ; Q_{e}$ the atomic electronic partition function; $E_{l}$ the energy of the atomic level 1; $k$ Boltzmann's constant; and $T$ the flame temperature. Note that this relation may not be significant for atoms with level separation much larger than $k T$; however, it would be very important for atoms, such as $\mathrm{Ga}$, whose ground state is a fine-structure level. ${ }^{20} \mathrm{By}$ combining the above two equations, one can determine the free atom number density $n_{a}$ from the time-integrated LEI signal by the working relation

$$
n_{a}=\frac{(x-y)}{G e \sigma \ell \gamma z}\left[\frac{Q_{e}}{g_{1}} e^{\left(E_{1} / k T\right)}\right] \int_{0}^{r} V(t) \mathrm{d} t
$$

where $z=\left\{x\left[1-\exp \left(-y \tau_{l}\right)\right]-y\left[1-\exp \left(-x \tau_{l}\right)\right]\right\}$.

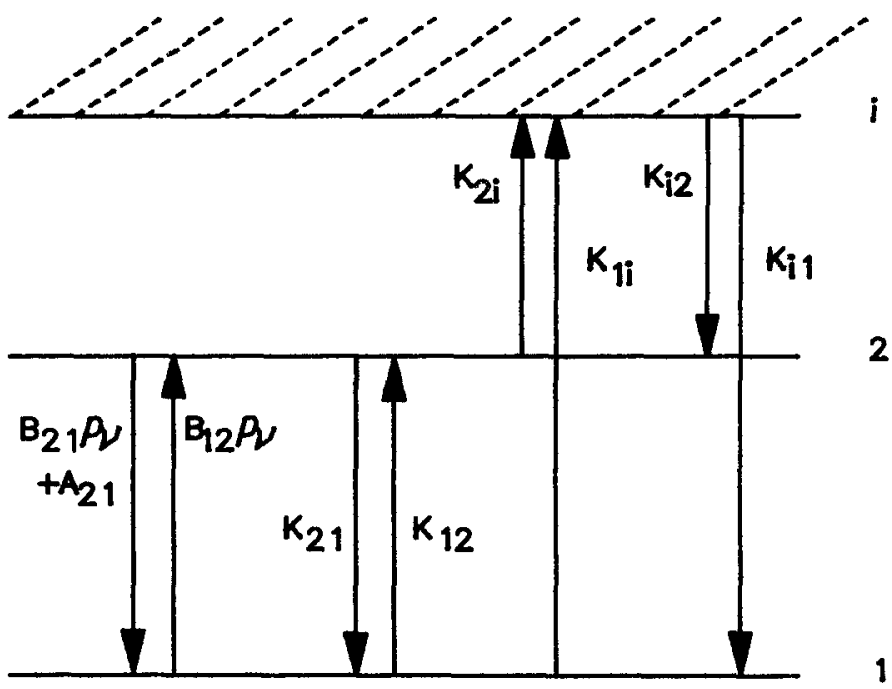

Fig. 1. Schematics for the three-level model for the LEI experiment.

The Total Number Density $n_{t}$. The total number density $n_{t}$ of the element in the flame is evaluated by the relation $^{8}$

$$
n_{t}=2.98 \times 10^{21} \frac{C \phi \epsilon}{\left(n_{T} / n_{298}\right) T f}
$$

where $C$ is the concentration (M) of the analyte solution; $\phi$ the aspiration rate $(\mathrm{cc} / \mathrm{min}) ; \epsilon$ the sample introduction efficiency; $T$ the flame temperature; $f$ the flow rate (cc/ s) of unburnt gases at room temperature $(298 \mathrm{~K}$ ) and atmospheric pressure; $n_{298}$ the number of moles of species at room temperature; and $n_{T}$ the number of moles of combustion products at temperature $T$.

Thus it is obvious that, if the Einstein $A$ and $B$ coefficients are available from the literature ${ }^{24}$ and the relevant rate coefficients are also given or can be properly estimated, one can determine the atomization efficiency of an element in a flame from the time-integrated LEI measurements via Eqs. 1, 13, and 14.

\section{EXPERIMENTAL}

The experimental setup for the LEI experiment is shown in Fig. 2. As was previously detailed, ${ }^{20}$ we used a commercial burner assembly (Perkin-Elmer) with a 100$\mathrm{mm} \times 0.5-\mathrm{mm}$ slot burner head, coupled with an interlocked gas control system by which acetylene $(0.5 \mathrm{~L} / \mathrm{min})$ and air $(12.5 \mathrm{~L} / \mathrm{min})$ were premixed prior to reaching the burner head. With the use of the formula of Chester et $a l .{ }^{25}$ the term $\left(n_{T} / n_{298}\right)$ in Eq. 14 was calculated to be 0.98 , corresponding to a complete reaction between acetylene and oxygen, as the lower limit for the case with an acetylene/air ratio of $1: 25$ and a flame temperature of

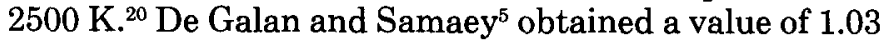
for the acetylene/air ratio of 1:9.83. Thus the real $\left(n_{T}\right)$ $n_{298}$ ) value should lie between 0.98 and 1.03 . We use the middle value 1.00 for simplicity. Aqueous solutions of each analyte were prepared at several concentrations. The aspiration rate was fixed at $4.5 \mathrm{cc} / \mathrm{min}$. The sample introduction efficiency $\epsilon$ was measured to be $0.088 \pm$ 0.005 . Using Eq. 14, we obtained the total number density $n_{t}$ of $(3.14 \pm 0.08) \times 10^{12},(1.57 \pm 0.04) \times 10^{12}$, and $(3.14$ 


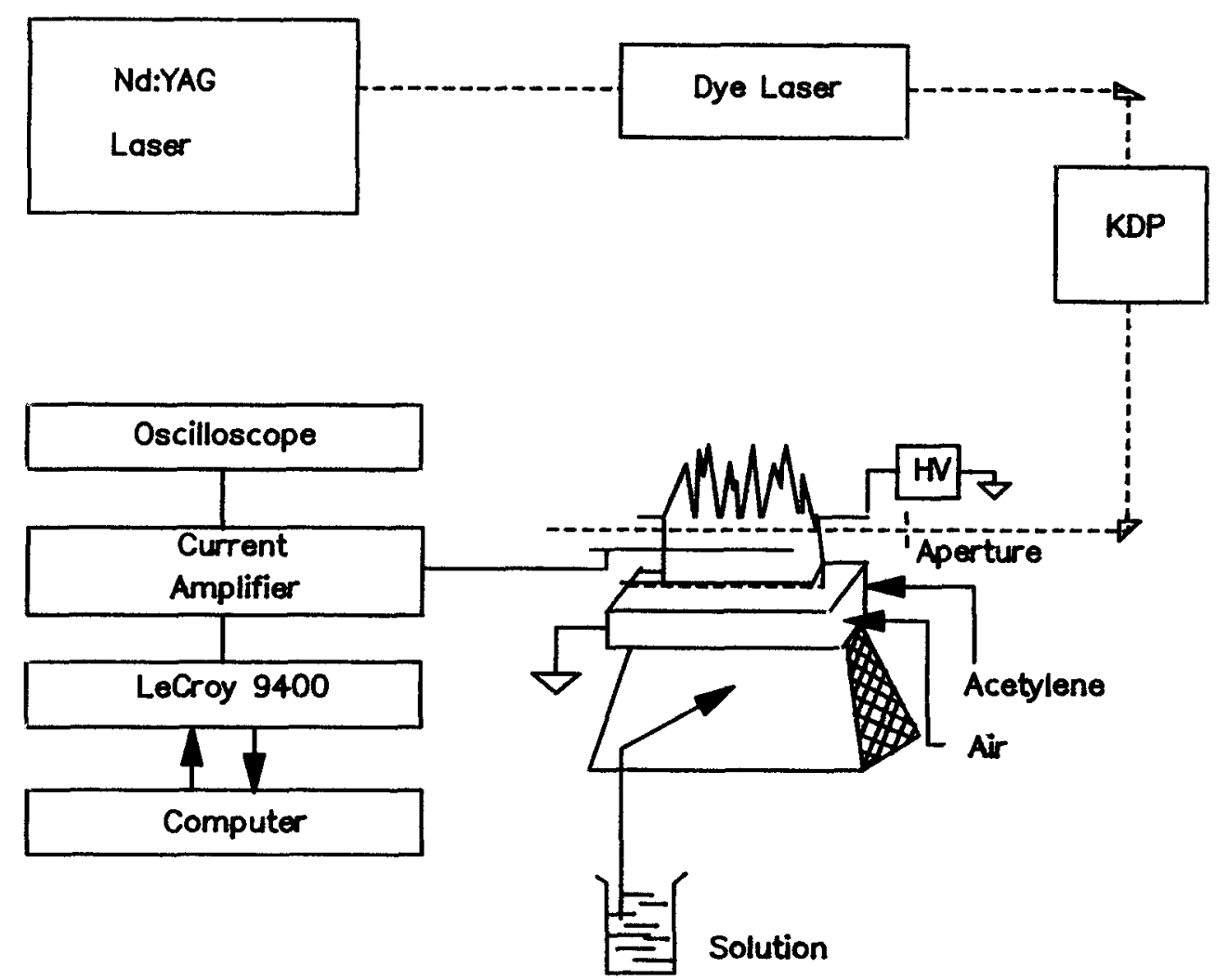

FIG. 2. Experimental setup for the time-integrated LEI measurement.

$\pm 0.08) \times 10^{11} \mathrm{~cm}^{-3}$ for lithium solutions of 10,5 , and 1 ppm, respectively, and of $(4.74 \pm 0.12) \times 10^{11} \mathrm{~cm}^{-3}$ for a sodium solution of $5 \mathrm{ppm}$.

A tunable dye laser (Quanta Ray PDL-2) pumped by a $10-\mathrm{Hz}$ frequency-doubled $\mathrm{Nd}$ :YAG laser (DCR-2A) was used. The dye laser had a pulse width of 5-8 ns and a linewidth of $\sim 1 \mathrm{~cm}^{-1}$. It was operated at a pulse energy, between 30 and $50 \mu \mathrm{J}$, which was monitored by a surface absorbing disk calorimeter (Scientech 36-0001). The la-

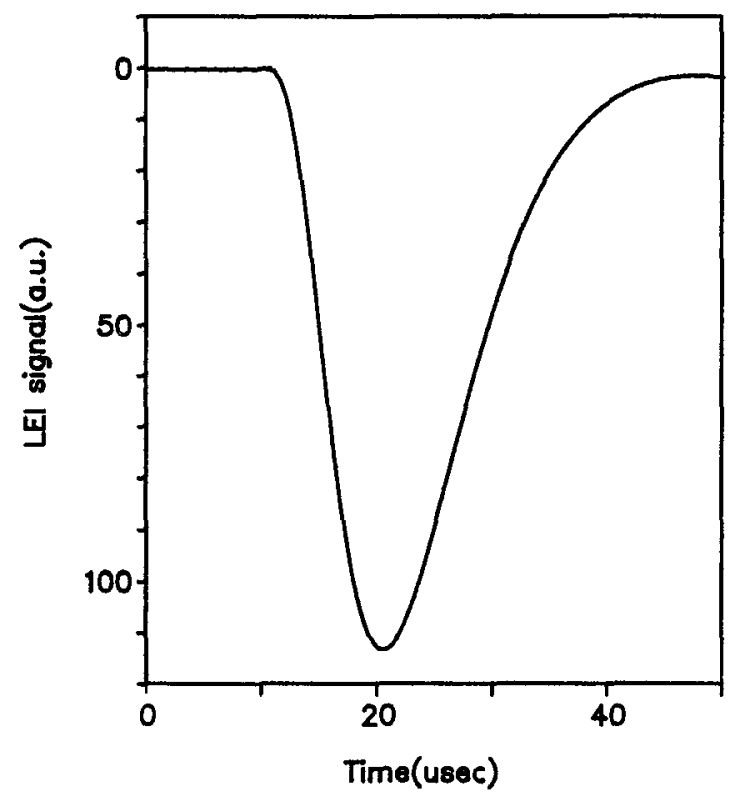

FIG. 3. One measured LEI voltage pulse due to the Li $2^{2} S_{1 / 2} \rightarrow 3^{2} P_{J}$ excitation. ser wavelength was precisely tuned to the line center of each applicable atomic transition through an LEI excitation spectrum scan. ${ }^{20}$ The laser beam was directed longitudinally through the flame at $7.5 \mathrm{~mm}$ above the burner head, and the beam size actually used was varied with an adjustable aperture. We will present the results obtained with a 2-mm-diameter laser beam.

The LEI current signal was probed either by a pair of 20-mm-long 1.5-mm-diameter stainless rod electrodes or by a pair of $20-\mathrm{mm} \times 16.5-\mathrm{mm} \times 0.5-\mathrm{mm}$ stainless plate electrodes, along the laser beam. The probing volume is $\sigma \ell=0.0628 \mathrm{~cm}^{3}$. The two electrodes were separated 12 $\mathrm{mm}$ apart and biased at a voltage of $500 \mathrm{~V}$. The detected signal was first amplified by a current amplifier (Keithley $427)$ with a calibrated gain factor $G=(5.0 \pm 0.2) \times 10^{4}$ V/A. The resultant voltage pulse was then recorded by a digital oscilloscope (LeCroy 9400) and stored on PC diskettes for later data treatment. One of the measured LEI voltage pulses due to the $\mathrm{Li} 2^{2} S_{1 / 2} \rightarrow 3^{2} P_{J}$ transition is shown in Fig. 3.

The ion collection efficiency $\gamma$ was determined in a least-squares manner by fitting the time-integrated LEI signal as a function of the distance of the collection electrode from the center of the laser beam, where a unity ion collection efficiency was assumed. The fitted plots for the case done with a 5-ppm sodium solution are presented in Fig. 4, in which the rod result was linearly fitted and the plate result could only be quadratically fitted. The reason for this discrepancy remains to be clarified. However, it is obvious that the LEI signal measured with the plate electrodes can increase more significantly than that with the rod electrodes, as the collector moves closer to the laser beam. The determined ion collection effi- 

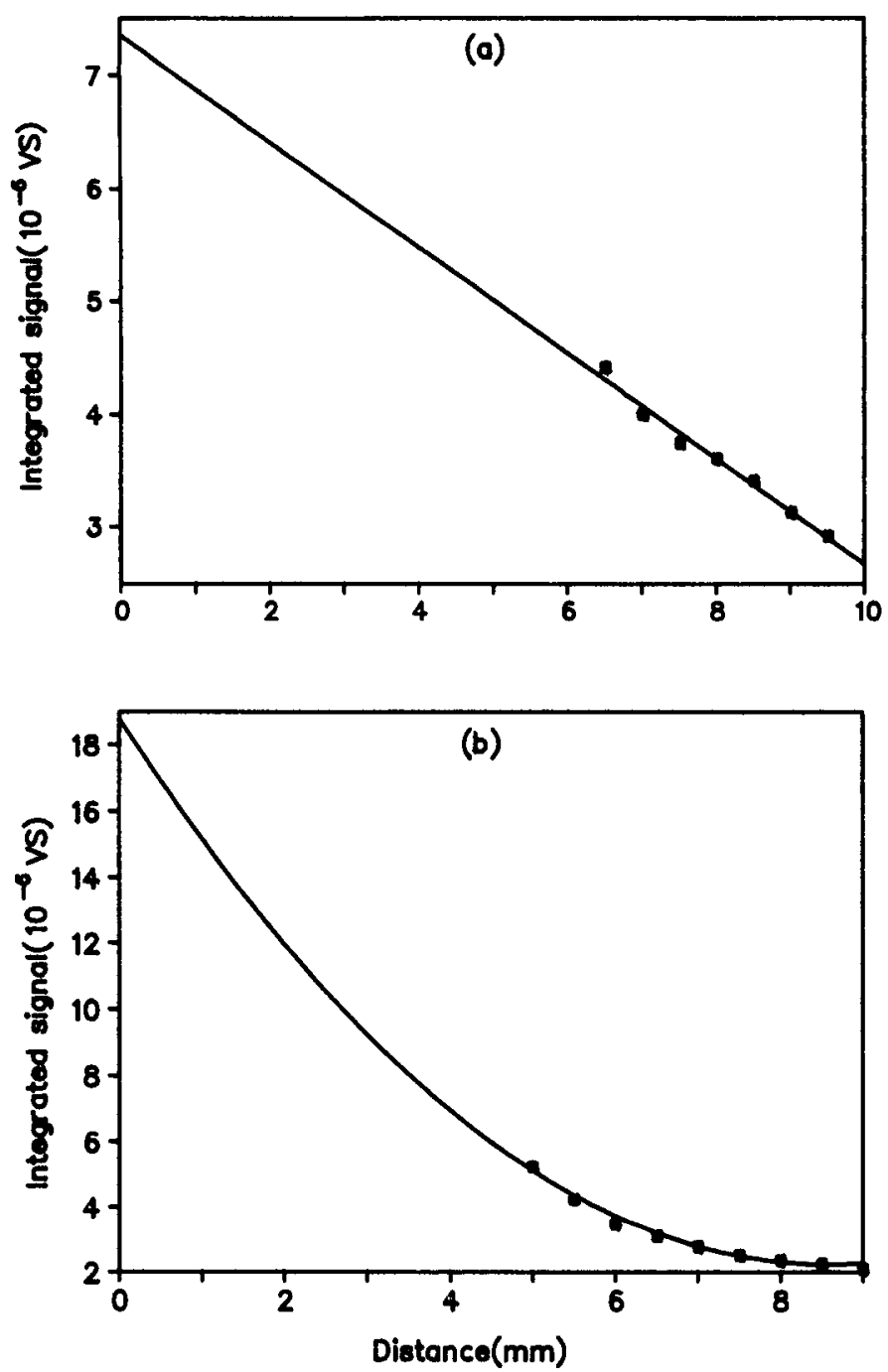

FIG. 4. Plots for the integrated LEI signal as a least-squares-fit function of the distance of the collection electrode from the center of the laser beam: (a) for the result detected with the rod electrodes, and (b) for the result detected with the plate electrodes.

ciencies are $0.48 \pm 0.03(0.16 \pm 0.03)$ for a $10-\mathrm{ppm}$ lithium solution, and $0.50 \pm 0.03(0.13 \pm 0.02)$ for a 5 -ppm sodium solution, where the first numbers are the results obtained with the rod electrodes and the parenthesized numbers are the results obtained with the plate electrodes.

\section{RESULTS AND DISCUSSION}

After integrating each measured LEI voltage pulse (see Fig. 3) over the time interval from 0 to $50 \mu \mathrm{s}$, and combining with the known electron charge, the amplifier gain factor, the probing volume, and the determined collection efficiencies, one can then determine the free atom number density $n_{a}$ using the relation in Eq. 13 . The involved atomic transitions, the Einstein $A$ coefficients, the energy and degeneracy of the level 1 , and the derived values of electronic partition functions of both $\mathrm{Li}$ and $\mathrm{Na}$ atoms are listed in Table I. All relevant collision rate coefficients, listed in Table II, are described below.

For the collision quenching rate coefficients $K_{21}$, we take into account only the quenching cross section due
TABLE I. The involved atomic transitions and Einstein coefficients of both $\mathrm{Li}$ and $\mathrm{Na}$ atoms, and parameters related to their electronic partition functions at $T=2500 \mathrm{~K}$.

\begin{tabular}{lcc}
\hline \multicolumn{1}{c}{ Atom } & \multicolumn{1}{c}{$\mathrm{Li}$} & $\mathrm{Na}$ \\
\hline Transition & $2^{2} S_{1 / 2} \rightarrow 3^{2} P_{J}$ & $3^{2} S_{1 / 2} \rightarrow 4^{2} P_{3 / 2}$ \\
$\lambda(\mathrm{nm})$ & 323.26 & 330.24 \\
$A_{21}\left(10^{6} \mathrm{~s}^{-1}\right)$ & $1.17^{\mathrm{a}}$ & $2.90^{\mathrm{b}}$ \\
$E_{1}\left(\mathrm{~cm}^{-1}\right)^{\mathrm{c}}$ & 0.0 & 0.0 \\
$g_{1}$ & 2 & 2 \\
$Q_{e}$ & 2.0011 & 2.0003 \\
$Q_{e} \exp \left(E_{1} / k T\right)$ & 1.00055 & 1.00015 \\
$g_{1}$ & &
\end{tabular}

a From W. L. Wiese, M. W. Smith, and B. M. Glennon, in Atomic Transition Probabilities, Vol. I, NSRDS-NBS 4 (1966). The uncertainty of the $A$ value is $10 \%$.

${ }^{b}$ From W. L. Wiese, M. W. Smith, and B. M. Miles, in Atomic Transition Probabilities, Vol. II, NSRDS-NBS 22 (1969). The uncertainty of the $A$ value is $25 \%$.

c All energies are relative to the ground level.

to the dominant collision with $N_{2}$. For the $\mathrm{Li} 3^{2} P_{J}$ state, we directly use the cross section of $21 \AA^{2}$, obtained by Jenkins ${ }^{26}$ for the Li $2^{2} P_{J}$ state, to calculate its quenching rate coefficient $K_{21}$. For the $\mathrm{Na} 4^{2} P_{3 / 2}$ state, we use the cross section of $43 \AA^{2}$, obtained by Gallagher et al., ${ }^{27}$ to evaluate its quenching rate coefficient $K_{21}$.

For the collision ionization rate coefficients $K_{2 i}$, we also consider only the dominant contribution from $\mathrm{N}_{2}$. Although no previous data are available, we may still reasonably estimate their values via the Hollander argument, ${ }^{28,29}$ which states that in a flame the collision ionization rate of an atom at its energy level $E_{\mathrm{j}}$ is proportional to both its statistical weight $g_{j}$ and its ionization probability, exp $\left|-\left(E_{\text {ion }}-E_{j}\right) / k T\right|$, where $E_{\text {ion }}$ is the ionization energy of the atom. Smyth et al. ${ }^{28}$ have applied this argument to the $\mathrm{Na} d$ states.

For the $\mathrm{Li} 3^{2} P_{J}$ state, we estimate its $K_{2 i}$ value to be $3.34 \times 10^{6} \mathrm{~s}^{-1}$ by using the $K_{3 i}$ value of $3.55 \times 10^{8} \mathrm{~s}^{-1}$ for the nearby $4 d$ states, reported recently by Smith et al. ${ }^{30}$ in a saturated LEI measurement. This also assumes that the degeneracy of the $3 p$ level is 6 , and the degeneracy of the $4 d$ state is 24 , and that there is the rapid mixing between the $4 d$ and $4 f$ states. $^{31}$ With this estimated $K_{2 i}$, we determine an $n_{\mathrm{a}}$ value of $(3.25 \pm 0.42) \times 10^{11} \mathrm{~cm}^{-3}$ of the $\mathrm{Li}$ free atom number density from the $10-\mathrm{ppm}$ lithium analyte solution. This result is consistent, at least within an order of magnitude, with the absorption result $n_{a} b=6.34 \times 10^{11} \mathrm{~cm}^{-2}$ of Smith et al., ${ }^{30}$ although a direct quantitative comparison is not possible since the laser

TABLE II. Some derived rate coefficients $\left(\mathrm{s}^{-1}\right)$ relevant for determining the free atom number density. The relations $B_{21}=\left(\lambda^{3} / 8 \pi h c\right) A_{21}$ and $B_{12}=\left(g_{2} / g_{1}\right) B_{21}$ are used to evaluate $B_{21}$ and $B_{12}$.

\begin{tabular}{llc}
\hline Atom & $\mathrm{Li}$ & $\mathrm{Na}$ \\
\hline$B_{21} \rho_{v}\left(10^{8}\right)$ & $3.88^{\mathrm{a}}$ & $12.0^{\mathrm{b}}$ \\
$B_{12} \rho_{v}\left(10^{9}\right)$ & 1.16 & 2.39 \\
$K_{21}\left(10^{7}\right)$ & 3.92 & 264.1 \\
$K_{2 i}\left(10^{6}\right)$ & 3.34 & 12.41 \\
$x\left(10^{9}\right)$ & 1.59 & 6.24 \\
$y\left(10^{6}\right)$ & 2.44 & 4.76 \\
$z\left(10^{7}\right)$ & 2.26 & 18.53 \\
\hline
\end{tabular}

a The average laser pulse energy at $323.26 \mathrm{~nm}$ is $30 \pm 1 \mu \mathrm{J}$, corresponding to a spectral energy density $\rho_{v}=1.63( \pm 0.06) \times 10^{-16} \mathrm{Jcm}^{-3} \mathrm{~Hz}^{-1}$.

${ }^{b}$ The average laser pulse energy at $330.24 \mathrm{~nm}$ is $35 \pm 1 \mu \mathrm{J}$, corresponding to a spectral energy density $\rho_{v}=1.90( \pm 0.04) \times 10^{-16} \mathrm{Jcm}^{-3} \mathrm{~Hz}^{-1}$. 


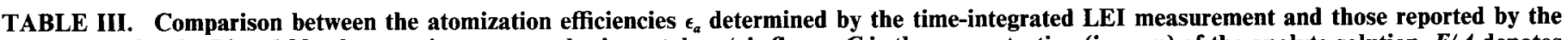

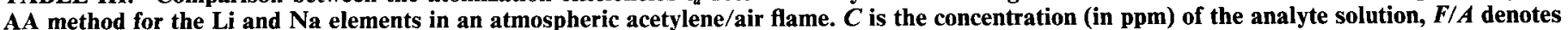
the flow rates (in $\mathrm{L} / \mathrm{min}$ ) of fuel and air, $T$ represents the flame temperature (in $K$ ).

\begin{tabular}{|c|c|c|c|c|c|}
\hline Atom & $\epsilon_{a}$ & $C$ & $F / A$ & $T$ & Ref. $^{\text {b }}$ \\
\hline $\mathrm{Li}$ & $\begin{array}{l}0.13 \pm 0.02(0.25 \pm 0.06)^{\mathrm{a}} \\
0.14 \pm 0.02(0.29 \pm 0.07) \\
0.16 \pm 0.02(0.37 \pm 0.09)\end{array}$ & $\begin{array}{r}10 \\
5 \\
1\end{array}$ & $0.5 / 12.5$ & 2500 & This work \\
\hline & $\begin{array}{l}0.21 \\
0.20 \\
0.12\end{array}$ & $\begin{array}{l}\cdots \\
32 \\
16.7\end{array}$ & $\begin{array}{c}0.7 / 7.56 \\
1 / 5.6 \\
0.96 / 9.44\end{array}$ & $\begin{array}{l}2480 \\
2450 \\
2450\end{array}$ & $\begin{array}{l}1 \\
2 \\
3^{*}\end{array}$ \\
\hline $\mathrm{Na}$ & $\begin{array}{c}1.0 \pm 0.1(1.0 \pm 0.2) \\
1.00 \\
0.50 \\
0.52 \\
1.00 \\
1.04\end{array}$ & $\begin{array}{l}5 \\
\cdots \\
0.1 \\
16 \\
0.05 \\
0.015\end{array}$ & $\begin{array}{c}0.5 / 12.5 \\
0.7 / 7.56 \\
1 / 5.6 \\
1 / 5.6 \\
1 / 5.6 \\
0.96 / 9.44\end{array}$ & $\begin{array}{l}2500 \\
2480 \\
2450 \\
2450 \\
2450 \\
2450\end{array}$ & $\begin{array}{l}\text { This work } \\
1 \\
2 \\
2 \\
2^{*} \\
3^{*}\end{array}$ \\
\hline
\end{tabular}

a The parenthesized values are the results from the plate electrodes.

b (1) From Ref. 32. (2) From Ref. $7\left[\left(^{*}\right)\right.$ measurement with 500 ppm Cs added]. (3) From Ref. 5 [(*) measurement with 1000 ppm Cs added].

interaction length $b$ was not given for the circular-shape flame they used. The $n_{a}$ values for 5-ppm and 1-ppm solutions are separately $(1.77 \pm 0.23) \times 10^{11} \mathrm{~cm}^{-3}$ and $(3.91 \pm 0.51) \times 10^{10} \mathrm{~cm}^{-3}$.

For the $\mathrm{Na} 4^{2} P_{3 / 2}(g=6)$ state, using the $\mathrm{Na} 3 d(g=$ 10) global ionization rate constant $K_{\text {ion }}$ value of $2.1 \times 10^{6}$ $\mathrm{s}^{-1}$ derived by Smyth et al. ${ }^{28}$ and also correcting the temperature to $2500 \mathrm{~K}$, following the same procedure we obtain a $\mathrm{K}_{2 i}$ value of $1.24 \times 10^{7} \mathrm{~s}^{-1}$ for the $\mathrm{Na} 4^{2} P_{3 / 2}$ state. With this derived $K_{2 i}$ value, we determine an $n_{a}$ value of $(3.80 \pm 0.40) \times 10^{11} \mathrm{~cm}^{-3}$ for the $\mathrm{Na}$ free atom number density from the 5-ppm sodium analyte solution.

Finally, we use Eq. 1 to determine the atomization efficiency of both lithium and sodium. The results are summarized in Table III, in which some previously reported values in an actylene/air flame, analyte concentrations, flow rates of fuel and air, and flame temperatures are also included for comparison. For the lithium atom case, the LEI results from the rod electrodes are quite consistent with those obtained by the atomic absorption spectroscopy, but the results from the plate electrodes are slightly higher. Note that, although the selected $\mathrm{Li}$ solutions are in the linear LEI response regime, the atomization efficiencies obtained with the solutions of lower $\mathrm{Li}$ concentrations (e.g., $1 \mathrm{ppm}$ ) appear to be slightly higher than those obtained with the 10-ppm Li solution, especially for the plate electrode case. We may attribute such a discrepancy to the fact that the assumption of uniform ion collection efficiency for all different concentrations of $\mathrm{Li}$ solutions may not be valid if there is an interference from the space charge effect. This interference could cause the collection efficiency determined at $10 \mathrm{ppm} \mathrm{Li}$ to be slightly smaller than its true value, and in turn cause the atomization efficiencies determined for the $\mathrm{Li}$ solutions of lower concentrations to be slightly higher.

For the sodium atom case, both the LEI result from the rod electrodes and that from the plate electrodes agree well with each other, and they are in excellent agreement with the AA value of Hinnov and Kohn (HK), ${ }^{32}$ and those AA values, determined with $\mathrm{Cs}$ added as a matrix element, of de Galan and Winefordner (DGW) ${ }^{7}$ and de Galan and Samaey. ${ }^{5}$ However our results are twice as high as those DGW AA values determined without Cs added. This significant discrepancy deserves some further discussion, although a full explanation is impossible at present. Previous studies have revealed that the flame temperature, ${ }^{5}$ the matrix element added, ${ }^{7}$ and the fuel/ oxidant ratio ${ }^{5}$ play important roles with regard to the degree of atomization of a salt solution. As shown in Table III, since there is just $50 \mathrm{~K}$ difference between the flame temperature of $2500 \mathrm{~K}$ in this work and the DGW value of $2450 \mathrm{~K}$, the above discrepancy is not likely to be attributed to the temperature factor. Given the flow rates of two flame gases, and the aspiration rate and the introduction efficiency of the analyte solution of this work, the use of the Saha equation ${ }^{33}$ provides an estimate of the number density of sodium ion $\left(\mathrm{Na}^{+}\right)$that is only $\sim 10 \%$ of that of free atoms of sodium. Thus it is possible that, in our case, most of sodium element may exist in the form of free atoms and, in the DGW case, one-half of the sodium element would exist in forms of ions and compounds. This argument is reasonable if we consider the difference between the acetylene/air ratio of 1:25 in this work and that of 1:5.6 in the DGW case. The DGW flame is a fuel-rich flame in which an extra amount of carbon-containing radicals can exist, affecting the atomization of sodium ${ }^{5}$ and further explaining why the Csmatrix effect comes to play an important role. Note that de Galan and Winefordner ${ }^{7}$ did also point out that the $\epsilon_{a}$ values changed little with the variation of the fuel/ oxidant ratio, but it is not clear to what extent the authors varied the fuel/oxidant ratio. On the other hand, our $\epsilon_{a}$ value is in excellent agreement with that of the HK case without matrix element added, where the acetylene/air ratio of 1:10.8 is only about one-half that of the DGW case. The above considerations seem to indicate that the discrepancy is most likely related to the difference in flame composition. However, to fully understand the detailed mechanism behind this discrepancy, more studies are definitely desirable. From the above results for both lithium and sodium, one can finally conclude that our LEI measurements agree well with those AA measurements, that our estimates on both collision quenching and collision ionization rate coefficients are quite appropriate, and that the LEI technique can indeed be used to determine the efficiency of the atomization of metal elements in a flame. 
In summary, we have demonstrated both theoretically and experimentally that the LEI technique can be used to determine the efficiency of the atomization of an element in an acetylene/air flame provided that the critical collision quenching and ionization rate coefficients are known or can be properly estimated. We have derived a useful relation between the time-integrated LEI signal and the total free atom number density in a flame. We have used the $\mathrm{Li}$ and $\mathrm{Na}$ analytes for the LEI test, and the resulting atomization efficiencies agree well with previously reported AA values.

\section{ACKNOWLEDGMENT}

This work is in part supported by the National Science Council of the Republic of China. The authors also thank the referee for valuable comments.

1. C. Th. J. Alkemade, T. Hollander, W. Snelleman, and P. J. Th. Zeegers, Metal Vapours in Flames (Pergamon Press, New York, 1982).

2. A. A. Pupyshev, N. I. Moskalenko, V. N. Muzgin, and Yu. S. Shalkauskas, J. Anal. Chem. USSR 45, 1641 (1991).

3. V. A. Osipova, Yu. Ya. Kuzyakov, K. A. Semenenko, and M. N. Gorlova, J. Anal. Chem. USSR 40, 630 (1985).

4. G. V. Kozyreva, S. L. Shcherbakova, and Yu. Ya. Kuzyakov, J. Anal. Chem. USSR 37, 916 (1982).

5. L. de Galan and G. F. Samaey, Spectrochim. Acta 25B, 245 (1970).

6. P. J. Th. Zeegers, W. P. Townsend, and J. D. Winefordner, Spectrochim. Acta 24B, 243 (1969).

7. L. de Galan and J. D. Winefordner, J. Quant. Spectrosc. Radiat. Transfer 7, 251 (1967).

8. J. D. Winefordner and T. J. Vickers, Anal. Chem. 36, 1939 (1964).

9. C. A. van Dijk, F. M. Curran, K. C. Lin, and S. R. Crouch, Anal. Chem. 53, 1275 (1981).

10. G. C. Turk, J. R. DeVoe, and J. C. Travis, Anal. Chem. 54, 643 (1982).

11. J. C. Travis, G. C. Turk, and R. B. Green, Anal. Chem. 54, 1006A (1982).
12. O. Axner, I. Lindgren, I. Magnusson, and H. Rubinsztein-Dunlop, Anal. Chem. 57, 773 (1985).

13. N. Omenetto, Th. Berthoud, P. Cavalli, and G. Rossi, Anal. Chem. 57,1256 (1985).

14. O. Axner, I. Magnusson, J. Peterson, and S. Sjostrom, Appl. Spectrosc. 41, 19 (1987).

15. K. C. Lin and Y. S. Duh, Appl. Spectrosc. 43, 20 (1989).

16. T. Berglind, S. Nillson, and H. Rubinstein-Dunlop, Phys. Scripta 36, 246 (1987).

17. K. S. Epler, T. C. O'Haver, G. C. Turk, and W. A. MacCreham, Anal. Chem. 60, 2062 (1988)

18. K. D. Su, S. H. Chou, K. C. Lin, and W. T. Luh, unpublished research.

19. K. C. Lin, P. M. Hunt, and S. R. Crouch, Chem. Phys. Lett. 90, 111 (1982).

20. K. D. Su, C. Y. Chen, K. C. Lin, and W. T. Luh, Appl. Spectrosc. 45, 1340 (1991).

21. J. C. Travis, J. Chem. Educ. 59, 909 (1982).

22. N. Omenetto, B. W. Smith, and L. P. Hart, Fresenius Z. Anal. Chem. 324, 683 (1986).

23. M. F. Hineman and S. R. Crouch, Spectrochim. Acta 43B, 1119 (1988).

24. W. L. Wiese and G. A. Martin, in Wavelengths and Transition Probabilities for Atoms and Atom Ions, Part II, NSRDS-NBS 68 (1980).

25. J. E. Chester, R. M. Dagnall, and M. R. G. Taylor, Anal. Chim. Acta 51, 95 (1970).

26. D. R. Jenkins, Proc. Roy. Soc. A306, 413 (1968).

27. T. F. Gallagher, W. E. Cooke, and S. A. Edelstein, Phys. Rev. A17, 125 (1978).

28. K. C. Smyth, P. K. Schenck, and W. G. Mallard, in Laser Probes for Combustion Chemistry, ACS Symp. Ser. No. 134, D. R. Crosley, Ed. (American Chemical Society, Washington, D.C., 1980), pp. 175181.

29. C. Th. J. Alkemade, T. Hollander, W. Snelleman, and P. J. Th. Zeegers, Metal Vapours in Flames (Pergamon Press, New York, 1982), p. 883

30. B. W. Smith, L. P. Hart, and N. Omenetto, Anal. Chem. 58, 2147 (1986).

31. L. M. Humphrey, T. F. Gallagher, W. E. Cooke, and S. A. Edelstein, Phys. Rev. A18, 1383 (1978).

32. E. Hinnov and H. Kohn, J. Opt. Osc. Am. 47, 156 (1957).

33. A. G. Gaydon and H. G. Wolfhard, Flames: Their Structure, Radiation, Temperature (John Wiley \& Sons, New York, 1978). 